\title{
Extension factor: Definition, properties and problems. Part 2
}

\author{
Krassimir T. Atanassov ${ }^{1}$ and József Sándor ${ }^{2}$ \\ ${ }^{1}$ Department of Bioinformatics and Mathematical Modelling \\ IBPhBME - Bulgarian Academy of Sciences, \\ Acad. G. Bonchev Str. Bl. 105, Sofia-1113, Bulgaria \\ and \\ Intelligent Systems Laboratory \\ Prof. Asen Zlatarov University, Bourgas-8010, Bulgaria \\ e-mail: krat@bas.bg \\ ${ }^{2}$ Babes-Bolyai University of Cluj, Romania \\ e-mails: jjsandor@hotmail.com, jsandor@math.ubbcluj.ro
}

Received: 1 September 2019

Accepted: 8 January 2020

Abstract: Some new properties of the arithmetic function called "Extension Factor" and introduced in Part 1 (see [5]) are studied.

Keywords: Arithmetic function, Extension factor.

2010 Mathematics Subject Classification: 11A25.

\section{Introduction}

In Part 1 of the present research (see [5]), we introduced the following arithmetic functions

$$
E F(n)=\prod_{i=1}^{k} p_{i}^{\alpha_{i}+1}
$$

that we called Extension Factor, where for the natural number $n=\prod_{i=1}^{k} p_{i}^{\alpha_{i}}: k, \alpha_{1}, \alpha_{2}, \ldots, \alpha_{k} \geq 1$ are natural numbers and $p_{1}, p_{2}, \ldots, p_{k}$ are different prime numbers. 
It is suitable to suppose that $p_{1}<p_{2}<\ldots<p_{k}$ and that $E F(1)=1$.

In the present paper, we will discuss other properties of function $E F$. In the text, we will use also the definitions of the following three well-known arithmetic functions (see [8]):

$$
\begin{aligned}
& \varphi(n)=\prod_{i=1}^{k} p_{i}^{\alpha_{i}-1} \cdot\left(p_{i}-1\right), \quad \varphi(1)=1 \quad \text { (Euler's totient function), } \\
& \psi(n)=\prod_{i=1}^{k} p_{i}^{\alpha_{i}-1} \cdot\left(p_{i}+1\right), \quad \psi(1)=1 \quad \text { (Dedekind's function), } \\
& \sigma(n)=\prod_{i=1}^{k} \frac{p_{i}^{\alpha_{i}+1}-1}{p_{i}-1}, \quad \sigma(1)=1 .
\end{aligned}
$$

We will use also the arithmetic functions (see $[1,8])$ :

$$
\begin{aligned}
\underline{\operatorname{mult}}(n) & =\prod_{i=1}^{k} p_{i}, \underline{\operatorname{mult}}(1)=1, \\
\Omega(n) & =\sum_{i=1}^{k} \alpha_{i}, \Omega(1)=1, \\
\delta(n) & =\sum_{i=1}^{k} \alpha_{i} p_{1}^{\alpha_{1}} \ldots p_{i-1}^{\alpha_{i-1}} p_{i}^{\alpha_{i}-1} p_{i+1}^{\alpha_{i+1}} \ldots p_{k}^{\alpha_{k}}, \\
\underline{\operatorname{set}(n)} & =\left\{p_{1}, p_{2}, \ldots, p_{k}\right\}, \\
\omega(n) & =k-\text { the cardinaliry of set } \underline{\operatorname{set}}(n) .
\end{aligned}
$$

It is immediate that, $\Omega(n) \geq \omega(n)$, with equality only if $n$ is a squarefree number, i.e. $n$ is a prime or a product of distinct primes.

\section{New properties of the arithmetic function $E F$}

Here, we will discuss some new properties of the arithmetic function $E F$.

Theorem 1. If $p$ is a prime number and $s<p$ is a natural number, then $E F(s)<E F(p)$.

Proof. Let $s<p$ be an arbitrary natural number. Then $E F(s)=s$.mult $(s) \leq s^{2}<p^{2}=$ $E F(p)$.

Corollary 1. Let $n>1$ be squarefree, written as $n=\prod_{i=1}^{k} p_{i}$. Then $E F(\varphi(n)) \leq E F(n)$.

Proof. We obtain sequentially that

$$
\begin{aligned}
E F(\varphi(n)) & =E F\left(\varphi\left(\prod_{i=1}^{k} p_{i}\right)\right)=E F\left(\prod_{i=1}^{k}\left(p_{i}-1\right)\right) \\
& \leq \prod_{i=1}^{k} E F\left(p_{i}-1\right) \leq \prod_{i=1}^{k} E F\left(p_{i}\right)=E F(n) .
\end{aligned}
$$


Theorem 2. For infinitely many $n$ one has $E F(\varphi(n))<E F(n)$, and for infinitely many $m$ one has $\operatorname{EF}(\varphi(m))>E F(m)$.

Proof. When $n=2^{k}$ for arbitrary natural number $k$, we obtain for $k=1$ :

$$
E F(\varphi(n))=E F(\varphi(2))=E F(1)=1<4=E F(2)=E F(n),
$$

and for $k \geq 2$ we obtain:

$$
E F(\varphi(n))=E F\left(\varphi\left(2^{k}\right)\right)=E F\left(2^{k-1}\right)=2^{k}<2^{k+1}=E F\left(2^{k}\right)=E F(n),
$$

but, when $n=3^{k}$, for $k=1$ :

$$
E F(\varphi(n))=E F(\varphi(3))=E F(2)=4<9=E F(3)=E F(n),
$$

and for $k \geq 2$ we obtain:

$$
E F(\varphi(n))=E F\left(\varphi\left(3^{k}\right)\right)=E F\left(2.3^{k-1}\right)=4.3^{k}>3^{k+1}=E F\left(3^{k}\right)=E F(n) .
$$

More generally, we can prove the following:

Theorem 3. For any odd prime $p$, and $k \geq 2$, for $m=p^{k}$, one has $E F(\varphi(m))>E F(m)$.

Proof. First, we see that for $k=1$ from Theorem 1:

$$
E F(\varphi(m))=E F(\varphi(p))=E F(p-1)<E F(p)=E F(m)
$$

and for $k \geq 2$ we obtain: $\varphi(m)=p^{k-1} \cdot(p-1)$, so $E F(\varphi(m))=p^{k} \cdot E F(p-1)$. Now,

$$
E F(p-1)=(p-1) \cdot \underline{\operatorname{mult}}(p-1) \geq 2 \cdot(p-1)
$$

as $\underline{\operatorname{mult}}(p-1) \geq 2$, because $p \geq 3$. Now, as $2(p-1)>p$, inequality (1) follows.

Theorem 4. Let $k, s \geq 1$ and $p \geq 3$ be an odd prime, satisfying $E F(p-1) \leq 2 p$. Let $n=2^{k} \cdot p^{s}$. Then one has

$$
E(\varphi(n))<E F(n)
$$

Proof. One has $\varphi(n)=2^{k-1} \cdot p^{s-1} \cdot(p-1)$, so using the inequality $E F(u \cdot v) \leq E F(u) \cdot E F(v)$, we can write

$$
E F(\varphi(n)) \leq 2^{k} \cdot p^{s} \cdot E F(p-1) \leq 2^{k+1} \cdot p^{s+1}=E F\left(2^{k} \cdot p^{s}\right)=E F(n),
$$

by using the assumption $E F(p-1) \leq 2 p$.

The cases $k=1$ and/or $s=1$ are checked as above. So, the inequality (2) holds. 
We must mention that examples of odd primes $p$, for which $E F(p-1) \leq 2 p$ are the following: $p=3,5,17$, and generally any Fermat prime $p=2^{r}+1$.

On the other hand, in the general case, between $E F(p)$ and, e.g., $E F(p+1)$ there is not a fixed relation, because, for example

$$
E F(8)=8 \times 2=16<49=7^{2}=E F(7),
$$

while

$$
E F(13)=13^{2}=169<196=14 \times 14=E F(14) .
$$

For this case, the following assertion is valid.

Theorem 5. If $p$ is a prime number and $p+1$ is squarefree, then

$$
E F(p)<E F(p+1) .
$$

If $p \geq 3$ is a prime number and $p+1$ is not squarefree, then

$$
E F(p)>E F(p+1) .
$$

Proof. First suppose that for the prime number $p, p+1$ is squarefree. If $p=2$, then

$$
E F(2)=4<9=E F(3)=E F(2+1) .
$$

If $p \geq 3$, then

$$
E F(p+1)=(p+1)^{2}>p^{2}=E F(p),
$$

i.e., the first case is valid.

If $p+1$ is not squarefree, then

$$
p+1=\prod_{i=1}^{k} p_{i}^{\alpha_{i}},
$$

where $k, \alpha_{1}, \alpha_{2}, \ldots, \alpha_{k} \geq 1$ are natural numbers and $p_{1}, p_{2}, \ldots, p_{k}$ are different prime numbers and there is at least one $i$ for which $\alpha_{i}>1$. Let $\alpha_{1}=2, p_{1}=2$ for $\alpha_{2}=\cdots=\alpha_{k}=1$. Then

$$
E F(p+1)=(p+1) \underline{\operatorname{mult}}(p+1)=\frac{(p+1)^{2}}{2}<p^{2}=E F(p),
$$

for $p \geq 3$. Obviously, if there is more than one $\alpha_{i}>1$, or if the smallest $p_{i}>2$, then the inequality will be more powerful.

Corollary 2. For every prime number $p \geq 3$ with $p+1$ squarefree,

$$
E F(\psi(n))>E F(n),
$$

while, otherwise, we have

$$
E F(\psi(n))<E F(n)
$$


From Theorem 1 it follows that for each prime number $p$ :

$$
E F(p)>E F(p-1),
$$

but there is not a fixed relation between $\operatorname{EF}(q)$ and $E F(p-1)$, where $q$ is the greatest prime number smaller than $p$, because, for example for $p=7, q=5$ : $E F(5)=25<36=E F(6)$, while for $p=17, q=13: E F(16)=32<169=E F(13)$.

It can be directly seen that for $n=1,2$,

$$
E F(n)^{n}=n^{E F(n)},
$$

because $E F(1)^{1}=1^{1}=1=1^{1}=1^{E F(1)}$ and $E F(2)^{2}=4^{2}=16=2^{4}=2^{E F(2)}$.

Theorem 6. For the natural number $n \geq 3: E F(n)^{n}<n^{E F(n)}$.

Proof. The proof follows directly from D. Mitrinović's inequality $(n+r)^{r}<n^{n+r}$ for the natural numbers $r$ and $n \geq 3$ [7].

\section{Function $E F$ and other arithmetic functions}

First, for a fixed natural number $n$ with the above canonical representation, we introduce the arithmetic functions

$$
I F(n)=\prod_{i=1}^{k} p_{i}^{\frac{1}{\alpha_{i}}}
$$

(see [2]),

$$
C F(n)=\prod_{i=1}^{k} \alpha_{i}^{p_{i}}
$$

(see [3]),

$$
R F(n)=\prod_{i=1}^{k} p_{i}^{\alpha_{i}-1}
$$

(see [4]), and well-known functions $\pi$, defined by $\pi(n)$ being the number of primes smaller or equal to $n$, and Möbius function $\mu$ (see [8]).

Obviously,

$$
\mu(E F(n))=0
$$

for each natural number $n>1$.

It is seen directly that for each natural number $n$ :

$$
\frac{E F(n)}{R F(n)}=\prod_{i=1}^{k} p_{i}^{1+\operatorname{sg}\left(\alpha_{i}-1\right)}=\underline{\operatorname{mult}}(n) \cdot \prod_{i=1}^{k} p_{i}^{\operatorname{sg}\left(\alpha_{i}-1\right)},
$$

where for each real number $x$ :

$$
\operatorname{sg}(x)=\left\{\begin{array}{l}
0, \text { if } x \leq 0 \\
1, \text { if } x>0
\end{array} .\right.
$$

Now, we prove the following assertions. 
Theorem 7. For every natural number $n$ : $I F(E F(n))<I F(n)$.

Proof. Let $n$ be a fixed natural number. Then

$$
I F(E F(n))=I F\left(\prod_{i=1}^{k} p_{i}^{\alpha_{i}+1}\right)=\prod_{i=1}^{k} p_{i}^{\frac{1}{\alpha_{i}+1}}<\prod_{i=1}^{k} p_{i}^{\frac{1}{\alpha_{i}}}=I F(n) .
$$

Theorem 8. For every natural number $n: C F(E F(n))>C F(n)$.

Proof. Let $n$ be a fixed natural number. Then

$$
C F(E F(n))=C F\left(\prod_{i=1}^{k} p_{i}^{\alpha_{i}+1}\right)=\prod_{i=1}^{k}\left(\alpha_{i}+1\right)^{p_{i}}>\prod_{i=1}^{k} p_{i}^{\alpha_{i}+1}=C F(n) .
$$

We check directly that

$$
\begin{aligned}
& E F(1)^{R F(1)}=1^{1}=1=1^{1}=R F(1)^{E F(1)}, \\
& E F(2)^{R F(2)}=4^{1}=4>1^{4}=R F(2)^{E F(2)}, \\
& E F(4)^{R F(4)}=8^{2}=64<256=2^{8}=R F(4)^{E F(4) .}
\end{aligned}
$$

More generally, the following assertion holds.

Theorem 9. For every squarefree natural number $n>1$ one has: $E F(n)^{R F(n)}>R F(n)^{E F(n)}$; and if $n$ is not squarefree, then $E F(n)^{R F(n)}<R F(n)^{E F(n)}$.

Proof. In the first case, we get $E F(n)^{R F(n)}>1=R F(n)^{E F(n)}$.

In the second one, we will discuss the simplest case, when $n=2^{k}$ for $k \geq 2$. The case $k=2$ was checked above, so, let $k \geq 3$. Then:

$$
\begin{gathered}
R F(n)^{E F(n)}-E F(n)^{R F(n)}=R F\left(2^{k}\right)^{E F\left(2^{k}\right)}-E F\left(2^{k}\right)^{R F\left(2^{k}\right)}=\left(2^{k-1}\right)^{2^{k+1}}-\left(2^{k+1}\right)^{2^{k-1}} \\
=2^{(k-1) 2^{k+1}}-2^{(k+1) 2^{k-1}}=2^{4(k-1) 2^{k-1}}-2^{(k+1) 2^{k-1}}>0,
\end{gathered}
$$

because $4(k-1)-(k+1)=3 k-5>0$ for each natural number $k \geq 1$. Obviously, in all other cases for $n$ the inequality will be more powerful.

Theorem 10. For every natural number $n$ not squarefree and with $\underline{\text { mult }}(n)>6$, it follows that $R F(n)^{E F(n)}>E F(n)^{n}$.

Proof. Let the natural number $n$ not squarefree be given, and let for brevity $r=\underline{\operatorname{mult}}(n)>6$.

We check sequentially that

$$
R F(n)^{E F(n)}-E F(n)^{n}=\left(\frac{n}{r}\right)^{n r}-(n r)^{n}>0,
$$

if and only if $n^{n r}-(n r)^{n} \cdot r^{n r}=n^{n r}-r^{n+n r} \cdot n^{n}=n^{n r}-r^{n(r+1)} \cdot n^{n}>0$, if and only if $n^{n(r-1)}-r^{n(r+1)}>0$.

Since $n$ is not squarefree, it follows that $n \geq 2 r$. Let us assume that $n=2 r$. Then

$$
\begin{gathered}
(2 r)^{2 r(r-1)}-r^{2 r(r+1)}=2^{2 r(r-1)} \cdot r^{2 r(r-1)}-r^{2 r(r+1)} \\
=4^{r(r-1)} \cdot r^{2 r(r-1)}-r^{2 r(r+1)}=r^{2 r(r-1)} \cdot\left(4^{r(r-1)}-r^{4 r}\right)>0,
\end{gathered}
$$

that is obvious valid for $r>6$. 
Theorem 11. For every odd not squarefree number $n$ one has: $R F(n)^{E F(n)}>E F(n)^{n}$.

Proof. Let the odd number $n$ be not squarefree. Then, as above, we must prove that $n^{n(r-1)}-r^{n(r+1)}>0$.

Now, clearly $n \geq 3 r$. Let us assume that $n=3 r$. Then

$$
\begin{gathered}
(3 r)^{3 r(r-1)}-r^{3 r(r+1)}=3^{3 r(r-1)} \cdot r^{3 r(r-1)}-r^{3 r(r+1)} \\
=r^{3 r(r-1)} \cdot\left(3^{3 r(r-1)} \cdot-r^{6 r}\right)>0
\end{gathered}
$$

because $3^{3 r(r-1)}$. $-r^{6 r}>0$ for $r \geq 3$ that is valid, because $n \geq 3$.

Corollary 3. For every squarefree natural number $n$

$$
n^{E F(n)}>E F(n)^{n}>E F(n)^{R F(n)}>R F(n)^{E F(n)} ;
$$

If $n$ is not squarefree, and $n$ is even, and $\underline{\operatorname{mult}}(n)>6$, or $n$ is an odd number, then

$$
n^{E F(n)}>R F(n)^{E F(n)}>E F(n)^{n}>E F(n)^{R F(n)} .
$$

Finally, we will discuss the relations between arithmetic functions $E F$ and $\pi$. In Table 1 we give the values for the first 20 natural numbers.

\begin{tabular}{|r|r|r|r|r|r|}
\hline$n$ & $\pi(n)$ & mult $(n)$ & $\pi($ mult $(n))$ & $E F(n)$ & $\pi(E F(n))$ \\
\hline 1 & 0 & 1 & 0 & 1 & 0 \\
2 & 1 & 2 & 1 & 4 & 2 \\
3 & 2 & 3 & 2 & 9 & 4 \\
4 & 2 & 2 & 1 & 8 & 4 \\
5 & 3 & 5 & 3 & 25 & 9 \\
6 & 3 & 6 & 3 & 36 & 11 \\
7 & 4 & 7 & 4 & 49 & 15 \\
8 & 4 & 2 & 1 & 16 & 6 \\
9 & 4 & 3 & 2 & 27 & 9 \\
10 & 4 & 10 & 4 & 100 & 25 \\
11 & 5 & 11 & 5 & 121 & 31 \\
12 & 5 & 6 & 3 & 72 & 20 \\
13 & 6 & 13 & 6 & 169 & 39 \\
14 & 6 & 13 & 6 & 196 & 44 \\
15 & 6 & 15 & 6 & 225 & 48 \\
16 & 6 & 2 & 1 & 32 & 11 \\
17 & 7 & 17 & 7 & 289 & 61 \\
18 & 7 & 6 & 3 & 108 & 30 \\
19 & 8 & 19 & 8 & 361 & 72 \\
20 & 8 & 10 & 4 & 200 & 46 \\
\hline
\end{tabular}

Table 1. Values of the functions for $n=1, \ldots, 20$. 
We are ready to formulate the following theorem.

Theorem 12. For each natural number $n \geq 8$ :

$$
\pi(E F(n)) \geq \pi(n) \cdot \pi(\underline{\operatorname{mult}}(n)) .
$$

Proof. In 1998, in [9], L. Panaitopol proved the inequality

$$
\pi(x) \cdot \pi(y)>\pi(x y)
$$

for any integers $x, y \geq 2$, excepting the following pairs: $(x, y)=(5,7),(7,5),(7,7)$.

Now, put $x=n, y=\underline{\operatorname{mult}}(n)$ in Panaitopol's inequality, to prove Theorem 12.

In fact, from the above, a slightly stronger form can be deduced, namely, it holds true for any $n \geq 2$ distinct from 7 .

Theorem 13. For each natural number $n \geq 2: \pi(E F(n)) \geq \pi(n)+\pi(\underline{\operatorname{mult}}(n))$.

Proof. In 1934 in [6], H. Ishikawa proved the inequality

$$
\pi(x y) \geq \pi(x)+\pi(y)
$$

for any integers $x, y \geq 2$. Now put $x=n, y=\underline{\operatorname{mult}}(n)$ in Ishikawa's inequality, to prove Theorem 13.

We can also state two results similar to Theorems 12 and 13, but involving the function $R F$ :

Theorem 14. For each natural number $n \geq 2$ :

$$
\pi(R F(n)) \leq \pi(n)-\pi(\underline{\text { mult }}(n))
$$

and

$$
\pi(R F(n)) \leq \frac{\pi(n)}{\pi(\underline{\operatorname{mult}}(n))}
$$

Proof. Apply the Ishikawa inequality $\pi(x y) \geq \pi(x)+\pi(y)$ for $x, y \geq 2$ for

$$
x=\underline{\operatorname{mult}}(n) \geq 2 \text { and } y=\frac{\pi(n)}{\pi(\underline{\operatorname{mult}}(n))} \geq 1 .
$$

Then we get the inequality (3).

We will remark, that (3) holds also for $x, y \geq 1$, since $\pi(1)=0$.

Now, for the proof of (3) apply the Panaitopol inequality $\pi(x y) \geq \pi(x) \pi(y)$ for

$$
x=\underline{\operatorname{mult}}(n) \text { and } y=\frac{\pi(n)}{\pi \underline{\operatorname{mult}}(n))} .
$$

Now the pairs

$$
\left(\underline{\operatorname{mult}}(n), \frac{\pi(n)}{\pi(\underline{\operatorname{mult}}(n))}\right)
$$

should be distinct from $(5,7),(7,5)$ and $(7,7)$. This is possible only if we do not have mult $(n)=7$ and $\frac{\pi(n)}{\pi(\underline{\operatorname{mult}}(n))}=7$, i.e., when $n=49$. Therefore, (4) follows. 


\section{Conclusion}

In conclusion, we will mention, that in future part we will study some extensions of the introduced new arithmetic function and some other functions.

\section{References}

[1] Atanassov K. (1987). New integer functions, related to $\varphi$ and $\sigma$ functions, Bulletin of Number Theory and Related Topics, XI (1), 3-26.

[2] Atanassov, K. (1996). Irrational factor: Definition, properties and problems. Notes on Number Theory and Discrete Mathematics, 2 (3), 42-44.

[3] Atanassov K. (2002). Converse factor: Definition, properties and problems, Notes on Number Theory and Discrete Mathematics, 8 (1), 37- 38.

[4] Atanassov K. (2002). Restrictive factor: Definition, properties and problems. Notes on Number Theory and Discrete Mathematics, 8 (4), 117-119.

[5] Atanassov, K. \& Sándor, J. (2019). Extension factor: Definition, properties and problems. Part 1. Notes on Number Theory and Discrete Mathematics, 25 (3), 36-43.

[6] Ishikawa, I. (1934). Über die Verteileung der Primzahlen, Sci. Rep. Tokyo Univ., 2, 21-44.

[7] Mitrinović, \& D., Popadić, M. (1978). Inequalities in Number Theory. Niś, Univ. of Niś.

[8] Mitrinović, D., Sándor, J. (in coop. with B. Crstici). (1995). Handbook of Number Theory, Kluwer Acad. Publ.

[9] Panaitopol, L. (1998). On the inequality $\pi(a) \cdot \pi(b)>\pi(a b)$. Bull. Math. Soc. Sci. Math. Roumanie, 41 (89), 2, 135-139. 